$R M x A C, \mathbf{5 3}, 166-168(2021)$

(c) 2021: Instituto de Astronomía, Universidad Nacional Autónoma de México

https://doi.org/10.22201/ia.14052059p.2021.53.34

\title{
THE CHANGING EVENT SURVEY: TRANSIENTS, SPACE DEBRIS \& NEOS
}

\author{
Tianrui Sun ${ }^{1,2}$, Chen Zhang ${ }^{1,2}$, Yiding Ping ${ }^{1,2}$, and Xuefeng $\mathrm{Wu}^{1,2}$
}

\section{RESUMEN}

Motivado por la prometedora Era de Astronomía del dominio temporal, y por la Astronomía de Multimensajeros, el proyecto CHanging Event Survey (CHES) está diseñado para unirse a la campaña en curso con un potente conjunto de telescopios ópticos de amplio campo. El proyecto CHES tiene como objetivo escudriñar el universo transitorio, incluyendo estallidos de rayos gamma, estallidos de radio rápidos, la contraparte electromagnética de los eventos de ondas gravitacionales (kilonova), supernovas, estrellas variables, objetos cercanos a la Tierra y basura espacial. La matriz de telescopios consta de 12 refractores individuales de gran campo y con apertura de $280 \mathrm{~mm}$, que cubren 600 grados cuadrados en total. En el ámbito del mismo proyecto, existen dos telescopios de $800 \mathrm{~mm}$ de diámetro que se pueden activar para hacer observaciones de seguimiento. Además, CHES puede monitorear efectivamente 300 grados cuadrados en una banda dual de manera simultánea, lo que permite que la matriz de telescopios realice la identificación y el seguimiento del candidato para en el marco de la colaboración LIGO / Virgo, y los satélites Fermi, Swift, GECam y SVOM.

\section{ABSTRACT}

Motivated by the promising era of time-domain and multi-messenger astronomy, CHanging Event Survey (CHES) is designed to join the ongoing campaign with a powerful wide-field optical telescope array. CHES project aims to monitor the transient universe, including gamma-ray bursts, fast radio bursts, the electromagnetic counterpart of gravitational waves events (kilonova), supernova, variable stars, near-earth objects, and space debris. The array consists of 12 individual wide field refractors with aperture $280 \mathrm{~mm}$, covering 600 square degrees in total. In the same project, two $800 \mathrm{~mm}$ prime focus telescopes can be triggered for follow-up observation. Furthermore, CHES can effectively monitor 300 square deg in a dual band simultaneously, which enable the array to do candidate identification and follow-up for the triggers from LIGO/Virgo collaboration, Fermi, Swift, GECam and SVOM satellites.

Key Words: gamma-ray burst: general — gravitational waves — methods: observational

\section{INTRODUCTION}

Time-domain astronomy requires fully automatic observational techniques and data reduction methods to process large amounts of images in real-time. In China, there're the Tsinghua-National Astronomical Observatories of Chinese Academy of Sciences Transient Survey (Zhang et al. 2015) and Purple Mountain Observatory-Tsinghua Supernova Survey.

Recently, we perform the CHanging Event Survey (CHES) for high cadence observations of transient and space debris in YaoAn Station. The CHES consist of the AllSky camera, the rapid survey array (twelve $280 \mathrm{~mm}$ telescopes) and the deep survey array (two $800 \mathrm{~mm}$ telescopes). All telescopes associated with CHES are listed in Table 1. YaoAn observation station is located in Yunnan

\footnotetext{
${ }^{1}$ Purple Mountain Observatory, Chinese Academy of Sciences, Nanjing 210033, China (xfwu@pmo.ac.cn).

${ }^{2}$ School of Astronomy and Space Science, University of Science and Technology of China, Hefei, Anhui 230026, China.
}

TABLE 1

TELESCOPES IN YAOAN SITE

\begin{tabular}{lrrc}
\hline Telescopes & Aperture & \multicolumn{1}{c}{ FoV } & MagLimit \\
\hline CHES28 & $280 \mathrm{~mm}$ & $7^{\circ} \times 7^{\circ}$ & 17.5 \\
CHES80 & $800 \mathrm{~mm}$ & $2^{\circ} \times 2^{\circ}$ & 20.5 \\
AllSky & $16 \mathrm{~mm}$ & $140 \mathrm{sq.} \mathrm{deg}$ & 12 \\
YAHPT & $800 \mathrm{~mm}$ & $10^{\prime} \times 10^{\prime}$ & 20.5 \\
\hline
\end{tabular}

Province $\left(101.1811^{\circ} \mathrm{E}, 25.5286^{\circ} \mathrm{N}\right)$ and belongs to Purple Mountain Observatory, Chinese Academy of Sciences.

For the rapid survey array, each of the $280 \mathrm{~mm}$ telescopes uses a $3056 \times 3056$ FLI CCD, and the pixel scale is $8.2^{\prime \prime}$. Thus we could detect sources brighter than 17.5 magnitudes within 60 seconds exposure and survey all the local sky above 25 degrees in 20 minutes. For the deep survey array, each of the $800 \mathrm{~mm}$ telescopes uses a $4108 \times 4096$ Andor CCD 


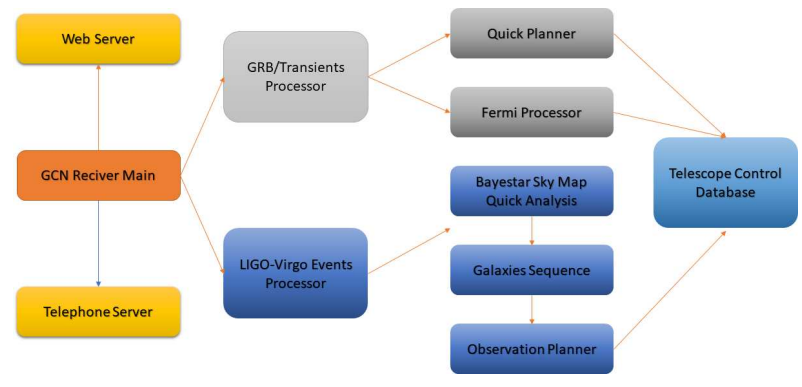

Fig. 1. Follow-Up Observation Strategy.

and the pixel scale is $1.7^{\prime \prime}$, the magnitude limit for $800 \mathrm{~mm}$ telescope in 60-second exposure is $20.5 \mathrm{~L}$ band magnitude in $5 \sigma$. All telescopes in both rapid and deep survey arrays have four filters, g, r, i and a very broad filter $\mathrm{L}$ with passband from $500 \mathrm{~nm}$ to $800 \mathrm{~nm}$.

In YaoAn station, another $800 \mathrm{~mm}$ aperture telescope, YaoAn High Precision Telescope (YAHPT) is a cooperative partner of CHES for observing specific sources and high precision photometry with Johnson passband UBVRI. The field of view (FoV) of YAHPT is $10^{\prime} \times 10^{\prime}$ with PIXIS $2048 \mathrm{~B}$ CCD in a pixel scale of $0.33^{\prime \prime}$ and limit to 20.5 magnitudes in $\mathrm{R}$ band in 60 seconds exposure.

\section{OBSERVATION STRATEGY FOR CHES}

Intentions of CHES are observing transients, near-earth objects, space debris and following up gamma-ray bursts, fast radio bursts, neutrino and gravitational wave events. To balance the observation time for different objects and optimize the quality of results, we designed different observation plans for CHES.

Regular observation is a composite survey for searching space debris and transients. We planned sky area lists for rapid and deep survey array as observation pointing sky areas. For each sky area, we take several templates in the moonless night with filter L, g, r, i. During observations, each sky area is observed in 10 seconds exposure for twelve times. Thus, the difference between each pair of images could be used to detect the space debris and the combined images could be utilized to explore the transients and near earth objects. CHES planner will generate observable sky areas and find the oldest observations as for observing.

Follow-up observations are based on the response of GCN notices and circulars. CHES has a planner which is a very complex and fully automatic strategy program for observation managements which is shown in Fig. 1. When the planner receives a required notice, it will automatically calculate the ob-

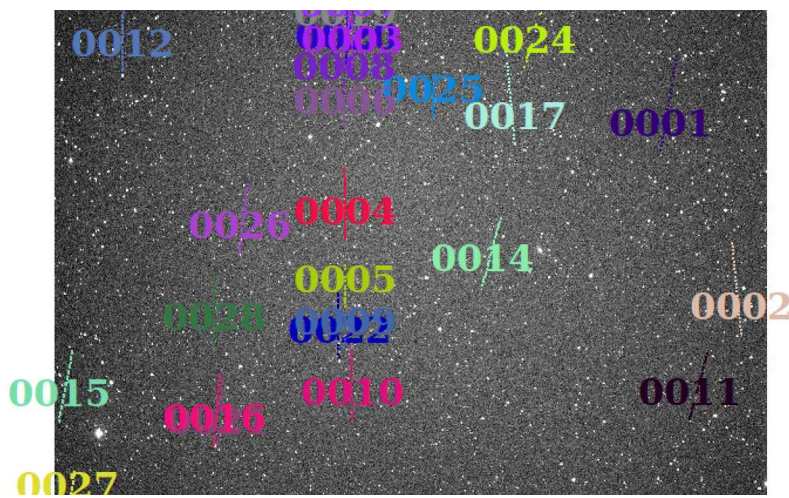

Fig. 2. Space Debris Example for Rapid Array.

servational possibilities and submit the observation requests to the SQL database with a higher priority. Ranks are determined by the error box of transient coordinates and types of notices. For the Swift triggers (Gehrels \& Swift Team 2004), the planner will only request one of the $800 \mathrm{~mm}$ telescopes for observations with gradually increased exposure time. As for Fermi satellite notices (Fermi Collaboration et al. 2009) and neutrino events notices, the $280 \mathrm{~mm}$ telescopes will be called to search transient, due to the large error box of positions of its receiver.

During April 2019 and May 2020, the O3 run of LIGO/Virgo Collaboration observation provides notices to observers by GCN networks. CHES planner can automatically download and analyze the Bayestar map (Singer et al. 2016) and further obtain the $50 \%$ possible sky areas according to the map and our templates pointings. Then, the planner will try optimize the pointing time, construct the observation sequence, and submit sequence to the database server with a higher rank for observations, as well.

\section{DATA ANALYSIS OF CHES}

The rapid/deep survey array will produce 400/100 GigaBytes of scientific data per day, respectively. A fast, stable and automatic pipeline system has been built to detect both space debris and transients from observed images. Astrometry is the first step of the pipeline for both space debris and transients. The optimized astrometry method (Ping et al. 2017) for a large field of view telescopes has been applied to the CHES system after each exposure. This method provides high precision world coordinates system parameters with SIP distortion in fourth-order (Shupe, D. L. et al. 2005).

In regular survey observations, the first step is to detect moving objects. CHES can detect several moving objects by comparing with catalogues from SExtractor (Bertin \& Arnouts 1996). Fig. 2 shows 


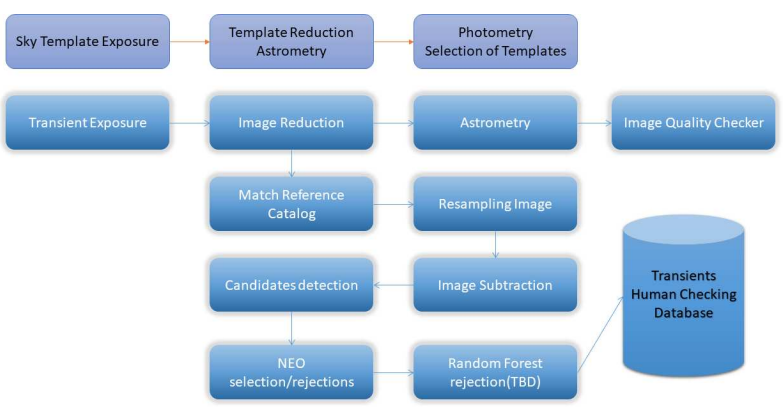

Fig. 3. Transient Detection Pipeline.

some detections in the FoV of the $280 \mathrm{~mm}$ telescope. After CCD reduction and median combining of a set of short-exposure images, the combined image is used for photometry and transient detection. For L band observations, GAIA DR2 catalogs can be considered as L band reference (Gaia Collaboration et al. 2018), while APASS can be referred in g, r, i bands (Henden et al. 2009).

In follow-up observations, observed images are processed by WCS calculations and CCD reductions directly. The initial step of photometry is using a simple configuration with MAG_AUTO to calculate median full-width half maximum, median major axis (A_IMAGE) and zero point difference from the reference. In aperture photometry, the aperture radius is calculated by six times the median major axis. Image registration is applied by the geometric methods with grmatch and fitrans in FITSH (Pál 2012).

We use local templates as the image subtraction reference for CHES and PanStarrs Data Release 1 images for YAHPT (Flewelling 2017). Transient detection uses image subtraction methods proposed by HOTPANTS (Alard \& Lupton 1998; Becker 2015). Detection of transients on subtracted images also utilizes SExtractor and filters by the shape of candidates and NEO positions from the database. The pipeline flow is described in Fig. 3.

CHES automatically observed GRB 191122A and LIGO/Virgo S191216ap with both 800mm telescope and $280 \mathrm{~mm}$ telescopes (Sun et al. 2019d,c). The observation for ZTF19abjethn and S190728 is shown in Fig. 4. The YAHPT also observed S190426a, S190425z and AT2019wxt for S191213g (Sun et al. 2019a,b,e).

\section{CONCLUSIONS}

In this paper, we introduce the CHanging Event Survey, a large FoV survey in YaoAn station. We successfully followed up some observable gamma-ray

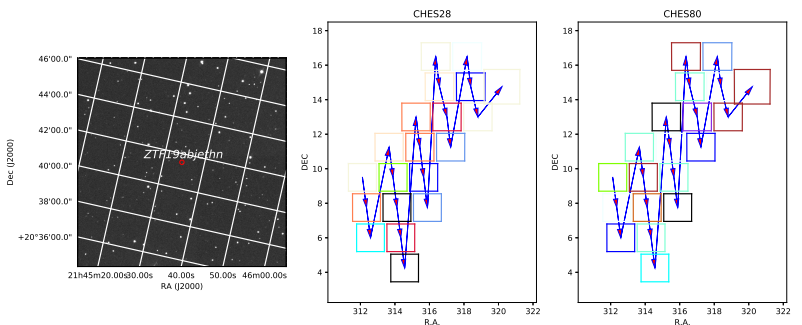

Fig. 4. Observation for S190728 candidates.

burst afterglows and searched several gravitational wave event high probability sky areas.

Acknowledgements: This research work was supported by the National Natural Science Foundation of China (Grant Nos.11603082, 11673070 and 11725314). We greatly appreciate to Center for Space Object and Debris Research of Chinese Academy of Sciences, and staffs in YaoAn station.

\section{REFERENCES}

Alard, C. \& Lupton, R. H. 1998, ApJ, 503, 325

Becker, A. 2015, HOTPANTS: High Order Transform of PSF ANd Template Subtraction, ascl:1504.004

Bertin, E. \& Arnouts, S. 1996, A\&AS, 117, 393

Fermi Collaboration, Giglietto, N., \& Fermi Collaboration 2009, NuPhS, 188, 273

Flewelling, H. 2017, AAS, 229, 237.07

Gaia Collaboration, Brown, A. G. A., Vallenari, A., et al. 2018, A\&A, 616, A1

Gehrels, N. \& Swift Team 2004, NewAR, 48, 431

Henden, A. A., Welch, D. L., Terrell, D., \& Levine, S. E. 2009, AAS, 214, 407.02

Pál, András. 2012, MNRAS, 421, 1825

Ping, Y. D., Zhang, C., \& Lu, C. L. 2017, AcASn, 58, 25

Shupe, D. L., Moshir, Mehdrdad, Li, J., et al. 2005, ASPC, 347, 491

Singer, L. P., Chen, H.-Y., Holz, D. E., et al. 2016, ApJS, 226,10

Sun, T., Chen, J., Hu, L., et al. 2019a, GCN, 24234 2019b, GCN, 24346

2019e, GCN, 26510

Sun, T., Zhang, C., Ping, Y., \& Wu, X. W. 2019c, GCN, 26271

2019d, GCN, 26487

Zhang, T. M., Wang, X. F., Chen, J. C., et al. 2015, RAA, 15, 215 